\title{
A short-term retrospective analysis of the clinical, histopathological and immunohistochemical aspects in endometrial malignancy
}

\author{
Tiberiu Augustin Georgescu ${ }^{1,2}$, Monica Cîrstoiu ${ }^{2,3 *}$, Mariana Costache ${ }^{1,2}$, Anca Lăzăroiu ${ }^{1,2}$, Adrian Dumitru ${ }^{1,2}$ and Maria Sajin ${ }^{1,2}$ \\ ${ }^{1}$ Department of Pathology, University Emergency Hospital Bucharest, Romania \\ 2"Carol Davila” University of Medicine and Pharmacy Bucharest, Romania \\ ${ }^{3}$ Department of Obstetrics-Gynecology, University Emergency Hospital Bucharest, Romania
}

\begin{abstract}
Endometrial carcinoma is the most frequent malignancy of the female reproductive system and the 7th cause of death by cancer in women from Western Europe. This study aims to evaluate the clinical, histopathological and immunohistochemical aspects as well as the short-term evolution in a series of 52 cases of endometrial carcinoma investigated, diagnosed and treated at the University Emergency Hospital in Bucharest, Romania over a period of one year. Demographic analysis revealed that in our country the majority of women presenting with endometrial carcinoma and, in particular, early stages of disease, usually come from urban regions and from areas with higher socioeconomic status. This raises the problem of disproportionate cultural levels and social inequity regarding access to healthcare services. All patients were postmenopausal and the mean age at presentation was 60.83, ranging from 43 to 81 years. Almost one-quarter (24\%) of the women who have undergone radical hysterectomy as treatment for endometrial carcinoma presented endometrial intraepithelial neoplasia (EIN) on a previous biopsy. Immunohistochemical comparison between EIN and EIN with concurrent endometrial carcinoma revealed that expression of PTEN was slightly higher in the first study group. Moreover, we were surprised to find that one of the 2 serous carcinomas presented diffuse estrogen receptor (ER) positivity within the papilary structure of the tumor, whereas the solid areas completely lost positivity for estrogen receptor (ER). Precise evaluation of the tumor type in endometrial cancers is one of the most frequent issues encountered in endometrial biopsies. Accurate diagnosis and distinction between type I and type II endometrial carcinoma is of critical importance for proper surgical planning and further clinical management.
\end{abstract}

\section{Introduction}

Endometrial carcinomas are a heterogeneous group of tumors originating from endometrial glandular epithelial cells, which represent the most frequent malignancies of the female reproductive system [1] and the $7^{\text {th }}$ cause of death by cancer in women from Western Europe (1-2\% of all deaths from cancer) [2].

In Romania, endometrial carcinoma has a peak incidence in the 60-64 age group, reaching an average value of 8.06/100,000 women in general population and 15.97/100,000 women within the highest risk age group. The estimated lifetime risk for a woman to develop endometrial cancer is approximately $1,03 \%$ [3]. The prevalence of endometrial carcinoma is presently recording an increasing trend worldwide as well as in Romania, mainly due to the rigorous and effective application of the screening methods and prophylaxis for cervical cancer.

Due to its early symptoms manifesting predominantly as abnormal vaginal bleeding, endometrial carcinoma is usually detected in its primary stages of disease, having good prognosis and a relatively high five-year survival rate. However, a significant percentage of patients are asymptomatic and have considerably reduced lifetime expectancy due to being diagnosed in advanced stages of disease [4,5].

\section{Materials and methods}

This retrospective analysis aims to evaluate the clinical, histopathological and immunohistochemical aspects as well as the short-term evolution of endometrial carcinoma in a series of cases investigated, diagnosed and treated at the University Emergency Hospital in Bucharest, Romania.

This study was elaborated as a descriptive-quantitative analysis performed over a period of one year (December 2014 - December 2015) and includes 52 cases of female patients histopathologically diagnosed with endometrial carcinoma in the Department of Pathology of the University Emergency Hospital in Bucharest, Romania.

Our digital hospital database facilitated the thorough investigation of each particular case with its clinical setting, medical history, prior investigations, diagnoses and treatments, all performed within their respective departments of our clinic. Data collection and processing was performed using Microsoft Office Excel 2010.

Biological samples obtained from endometrial biopsies or hysterectomy specimens have been processed using the conventional method of paraffin embedding and hematoxylin and eosin staining. Tissue samples were initially fixed for 24 hours in $10 \%$ buffered

Correspondence to: Monica Cirstoiu, Lecturer, MD, $\mathrm{PhD}$, Department of Obstetrics-Gynecology, Emergency University Hospital, 169 Independenței Avenue, 050098 Bucharest, Romania, E-mail: dr_cirstoiumonica@yahoo.com

Key words: endometrial carcinoma, endometrial endometrioid carcinoma, endometrial serous carcinoma

Received: December 18, 2015; Accepted: January 08, 2016; Published: January 11,2016 
formalin and afterwards processed and embedded in paraffin. Hematoxylin and eosin staining was performed on three-micron thick sections cut from these blocks. Immunohistochemical staining was performed on fresh paraffin sections using an indirect tristadial Avidin-Biotin complex method. Sections were first deparaffinated in toluene, dehydrated in alcohol series, then rehydrated and washed in phosphate buffered saline. Afterwards, they were incubated with primary antibody overnight, washed with carbonate buffer and developed in 3,3'-diaminobenzidine hydrochloride/hydrogen peroxide nuclear counterstaining with Mayer's Hematoxylin. The following immunomarkers were used: Ki-67 antibody (Rabbit Monoclonal antibody, Biocare, dilution 1:100, clone SP6, catalog number CRM 325 A, B, C), ER - estrogen receptor (mouse monoclonal antibody cocktail produced by Biocare, dilution 1:100, clone 1D5, catalog number ACI 054 A,C), PR - progesterone receptor (mouse monoclonal antibody provided by Biocare, dilution 1:50, clone 16, catalog number ACA 424 A, C), PTEN Antibody (goat polyclonal antibody, provided by Novus, dilution $10 \mathrm{ug} / \mathrm{mL}$, catalog number AF6655), CEA antibody (rabbit polyclonal, Biocare, dilution 1:200, catalog number CP 009 A,B,C) and Vimentin (Rabbit Monoclonal antibody, Biocare, dilution 1:50, clone SP20, catalog number CRM $312 \mathrm{~A}, \mathrm{~B})$.

\section{Results}

As expected, clinical features of the studied population were unspecific. Most patients addressed the Department of ObstetricsGynecology of the University Emergency Hospital Bucharest for abnormal vaginal bleeding. A low number of women presented other diagnostic circumstances, including: various anomalies at cervical examination, accidental CT discovery of a thickened $(>4 \mathrm{~mm})$ post-menopausal endometrium, suggestive ultrasound aspects of endometrial hyperplasia or endometrial polyps.

Demographic analysis revealed that the vast majority of patients $(86,4 \%)$ came from urban areas. All patients were postmenopausal and the mean age at presentation was 60.83 , ranging from 43 to 81 years. Distribution of cases according to age groups is presented in Figure 1.

Hospital database queries revealed that 38 of the women who undergone radical hysterectomy had at least one endometrial biopsy in the past 12 months within our hospital unit. Among them, almost onequarter (24\%) presented endometrial intraepithelial neoplasia (EIN) on a previous biopsy. Previous biopsy findings in patients treated for endometrial carcinoma are presented in Figure 2.

Initial histopathological diagnosis was established predominantly

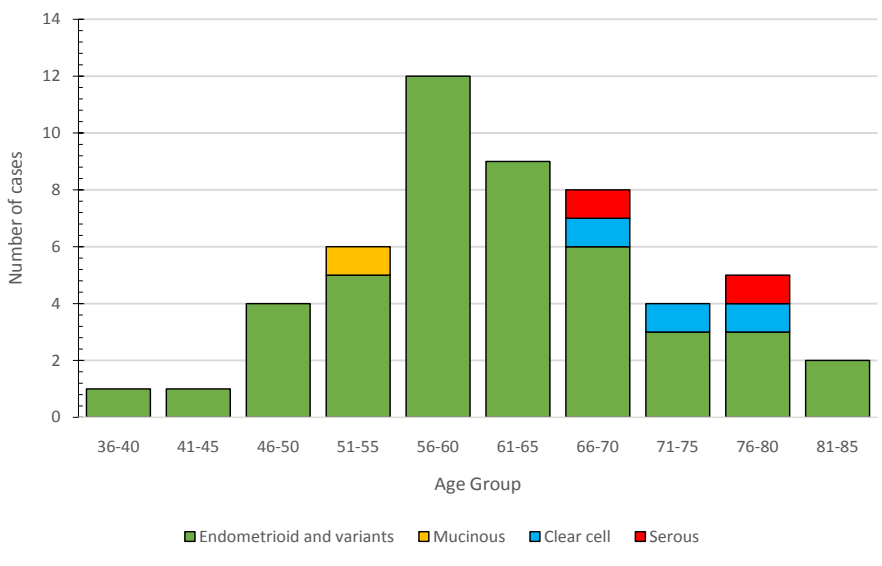

Figure 1. Distribution of cases according to age groups.

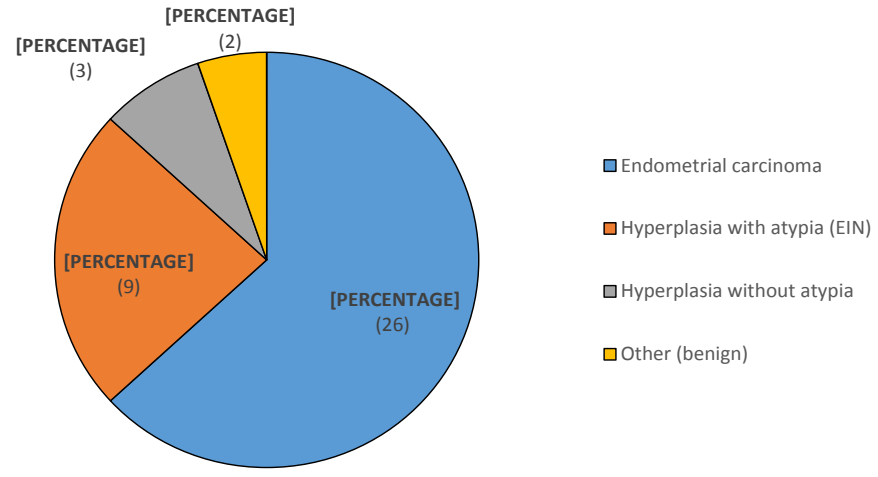

Figure 2. Previous biopsy findings in patients treated for endometrial carcinoma.

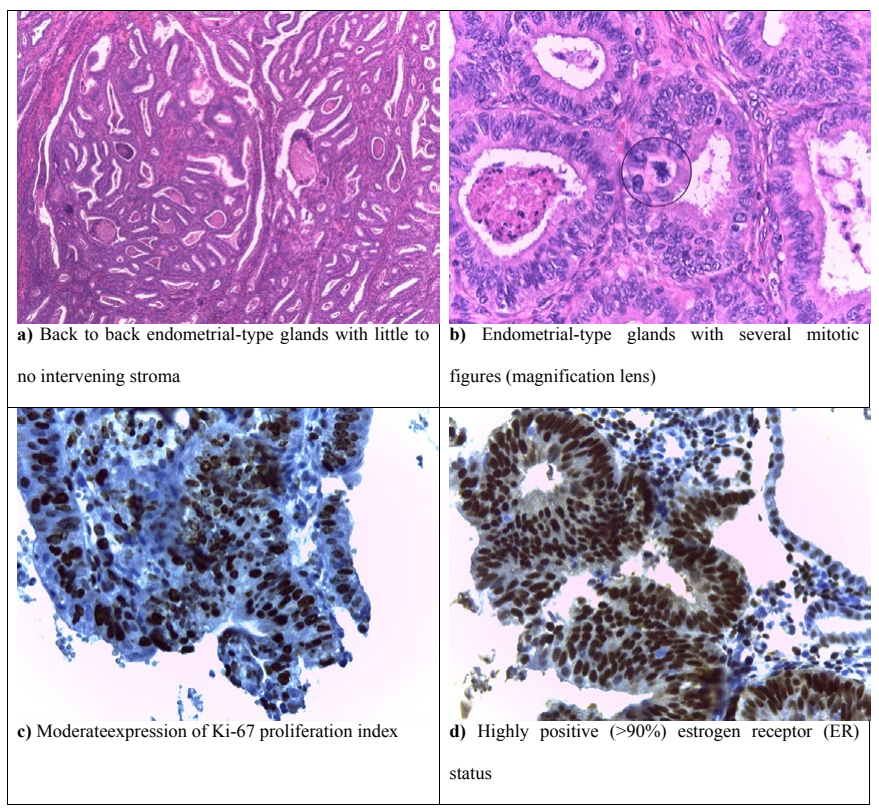

Figure 3. Well differentiated endometrial endometrioid carcinoma.

on endometrial biopsies. A few cases, which had previous investigations in other sanitary units, were diagnosed postoperatively directly on the hysterectomy specimen. All patients were suspected of endometrial malignancy and there were no incidental findings recorded. Most patients diagnosed with endometrial carcinoma were treated in our clinic by radical hysterectomy with bilateral salpingo-oophorectomy and regional lymph node excision. A minority of cases underwent the same type of treatment in other sanitary units. For patients treated in our clinic, only the hysterectomy specimen was included in the statistical analysis. Endometrial biopsies included in the study are from patients diagnosed in our clinic but treated in other sanitary units or from patients who were not yet treated at the time of this analysis.

Histopathological evaluation was performed on all 52 cases of endometrial carcinoma and involved the following criteria: architectural pattern, tumor differentiation and surgical staging. The most frequently encountered histopathological type of endometrial cancer was endometrioid carcinoma (46 cases), followed by clear cell carcinoma (3 cases), serous carcinoma ( 2 cases) and mucinous carcinoma ( 1 case). Among the 46 cases of endometrial endometrioid carcinoma, 24 cases were of the usual type (Figure 3), 12 cases presented villo-glandular pattern and 10 cases revealed squamous 
differentiation. Less frequently encountered histopatological types of endometrial cancer included 3 cases of clear cell carcinoma, 2 cases of serous carcinoma (Figure 4) and 1 case of mucinous carcinoma. The frequency of each type of endometrial cancer identified in the studied population is presented in Table 1.

Histopathological analysis also revealed various types of endometrial metaplasia. Squamous metaplasia was the most common type of epithelial change encountered predominantly in association with type I endometrioid carcinoma. Other types of epithelial change encountered were: eosinophilic change, ciliated metaplasia and clear cell change or secretory metaplasia.

Upon microscopic examination, most cases of endometrioid carcinoma revealed complex and extensive epithelial growth patterns forming back to back endometrial-type glands with little to no intervening stroma (Figure 3). Less differentiated endometrioid carcinomas were composed of sheet-like tumor cells with no glandular structures, prominent nuclear atypia, frequent angiolymphatic and deep myometrial invasion. Stroma was usually scant and desmoplastic with foamy cells and tumoral necrosis.

Both cases of serous carcinoma had a microscopically characteristic papillary structure composed of papillae varying from short, branching and hyalinized to long, thin and delicate, covered by exfoliative bulbous hobnail-like cells with marked nuclear atypia (Figure 4).

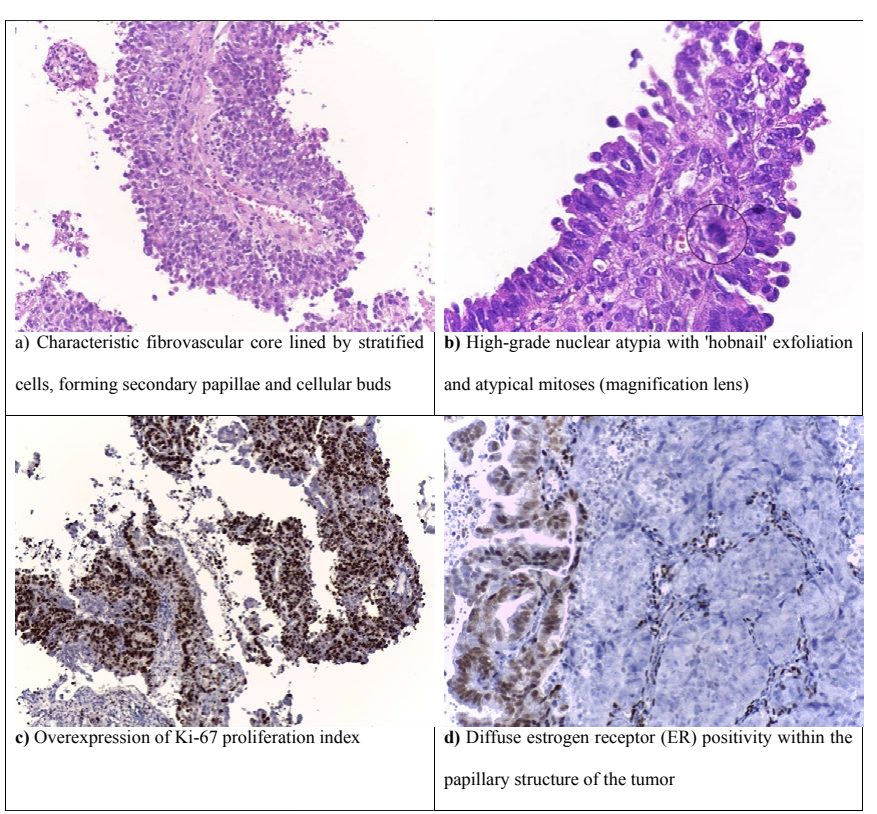

Figure 4. Endometrial carcinoma, serous type.

Table 1. Frequency of the histopathological diagnoses in the studied population.

\begin{tabular}{|c|c|c|c|}
\hline Type of lesion & Hysterectomy & $\begin{array}{c}\text { Endometrial } \\
\text { Biopsy }\end{array}$ & Total \\
\hline Endometrial Carcinoma & 44 & 8 & 52 \\
\hline - $\quad$ Endometrioid & 40 & 6 & 46 \\
\hline - of the usual type & 21 & 3 & 24 \\
\hline - $\quad$ villoglandular pattern & 10 & 2 & 12 \\
\hline - $\quad$ with squamous differentiation & 9 & 1 & 10 \\
\hline - $\quad$ Clear cell & 1 & 2 & 3 \\
\hline - $\quad$ Serous & 1 & 1 & 2 \\
\hline - $\quad$ Mucinous & 1 & 0 & 1 \\
\hline
\end{tabular}

Table 2. Tumor differentiation and surgical staging of the 46 cases of endometrial endometrioid carcinoma included in the study.

\begin{tabular}{|c|c|c|c|c|}
\hline \multicolumn{2}{|c|}{ GRADING } & & \multicolumn{2}{c|}{ STAGING } \\
\hline G1 & G2 & G3 & No. of patients & FIGO Stage \\
\hline 7 & 2 & 0 & 9 & IA \\
\hline 7 & 4 & 1 & 12 & IB \\
\hline 6 & 4 & 0 & 10 & II \\
\hline 1 & 3 & 0 & 4 & IIIA \\
\hline 0 & 0 & 0 & 0 & IIIB \\
\hline 1 & 0 & 2 & 3 & IIIC1 \\
\hline 0 & 1 & 1 & 2 & IIIC2 \\
\hline 0 & 0 & 0 & 0 & IVA \\
\hline $\mathbf{2 2}$ & 0 & 0 & 0 & IVB \\
\hline & $\mathbf{1 4}$ & $\mathbf{4}$ & $\mathbf{4 0}$ & $\begin{array}{c}\text { Total } \\
\text { hysterectomy } \\
\text { specimens }\end{array}$ \\
\hline $\mathbf{1}$ & & & & $\begin{array}{c}\text { Total } \\
\text { endometrial } \\
\text { biopsies }\end{array}$ \\
\hline
\end{tabular}

Tumor differentiation based on the amount of solid growth of the glandular component (Grade I <5\%; Grade II 5-50\%; Grade III >50\%) was established in all cases of endometrial endometrioid carcinoma and is presented in Table 2. Most tumors were highly differentiated, less invasive and therefore associated with early stages of disease. Moderately and undifferentiated tumors were fewer, more invasive and usually associated with higher stages of disease. Surgical staging was performed in all cases of endometrial endometrioid carcinoma treated by hysterectomy with bilateral salpingo-oophorectomy and regional lymph node excision and is also presented in Table 2.

In order to determine whether immunohistochemical markers in endometrial intraepithelial neoplasia could predict the presence of a concurrent endometrial carcinoma we established 3 immunohistochemical study groups. The first immunohistochemical study group involved samples from all 52 cases of endometrial carcinoma. The second immunohistochemical study group included samples from the initial endometrial biopsies of the 9 cases of endometrial carcinoma which were preoperatively diagnosed with endometrial intraepithelial neoplasia. The third immunohistochemical control group was composed of biopsy samples from 10 cases of endometrial intraepithelial neoplasia diagnosed and treated in our clinic, in which the diagnosis of EIN was confirmed on the hysterectomy specimen and no concurrent invasive carcinoma has been found.

Ki-67 proliferation index showed great variability among all examined cases. Type I endometrioid carcinomas recorded the smallest values with progressive increase from low-grade and well-differentiated tumors to high-grade and undifferentiated tumors. Overexpression was associated with type II non-endometrioid carcinomas as well as high-grade and highly invasive tumors.

Highly positive (>90\%) estrogen and progesterone receptor status (ER/PR) was usually associated with well-differentiated carcinomas, small depth of myometrial invasion and absence of regional lymph node metastases.

We were surprised to find that one of the 2 serous carcinomas presented diffuse estrogen receptor (ER) positivity within the papilary structure of the tumor, whereas the solid areas completely lost positivity for estrogen receptor (ER). This is probably due to the intratumoral heterogeneity of high grade tumors. 
Other immunomarkers with low specificity have been tested as well: Vimentin was positive in all cases of endometrioid carcinoma while CEA positivity was present in a reduced number of cases, being largely confined to squamous areas with only 6 specimens exhibiting focal membranous staining of the glandular component.

Comparison of the second and third immunohistochemical study groups revealed that expression of PTEN was slightly higher in endometrial intraepithelial neoplasia than in concurrent EIN and endometrial carcinoma.

\section{Discussion}

Endometrial carcinoma encompasses a large spectrum of neoplasms with variable histologic differentiation that ranges from well-differentiated to solid and poorly differentiated carcinomas. For decades, this was the main classification criteria for endometrial carcinomas [6]. After serous carcinoma was identified in 1982 [7], it was soon acknowledged as the most aggressive type of endometrial cancer, unrelated to estrogenic stimulation. Therefore, the need for a more elaborate and clinically predictive classification system has led to the separation of endometrial carcinomas in two distinct groups designated as type I (endometrioid) and type II (non-endometrioid) endometrial carcinoma [8]. Type I endometrial carcinomas develop in perimenopausal women in the setting of hyperestrogenism. These are usually low-grade tumors without deep myometrial invasion which usually coexist or are preceded by endometrial intraepithelial neoplasia (EIN). Type II endometrial carcinomas are very aggressive neoplasms, unrelated to estrogenic stimulation which usually appear in postmenopausal, elderly women. Those are usually high-grade and deeply-invasive tumors which are not preceded or associated with EIN.

The most significant risk factor for type I endometrial carcinoma is long-term exposure to endogenous or exogenous estrogens, unopposed by progestins. The relative risk of developing endometrial adenocarcinoma in women taking unopposed estrogens is increased by 3 - to 6-fold [9], rising to 9.5 fold if unopposed estrogen has been used for more than 10 years [10]. Progesterone has long been known as having a protective effect on the endometrium, reducing the incidence of endometrial carcinoma when used by young women in oral contraceptives $[11,12]$. Other risk factors include histologic type and grade, tumor stage, depth of myometrial invasion, lymphovascular involvement, age at diagnosis and steroid hormone receptor status. Tamoxifen therapy is also associated with an overall increased risk of endometrial carcinoma [13]. Moreover, women treated with tamoxifen are more likely to develop endometrial polyps, especially ones of gigantic size [14].

The 2 systems used for staging endometrial carcinoma are the FIGO (International Federation of Gynecology and Obstetrics) system and the American Joint Committee on Cancer TNM staging system which is basically the same, the difference being that the FIGO system does not include stage 0 . Clear cell, serous and mucinous carcinomas have not been included in this classification. Clear cell and serous carcinomas are considered high grade tumors by definition, while mucinous carcinomas are almost always well differentiated neoplasms with relatively good prognosis [15]. Our findings confirm that certain histologic types of endometrial carcinoma are associated with better prognosis and higher overall survival rates. Type I endometrioid carcinoma and its variants are usually associated with a relatively good prognosis, while non-endometrioid type II carcinomas show unfavorable outcomes.
In Romania, most women presenting with endometrial carcinoma and, in particular, early stages of disease, usually come from urban regions and from areas with higher socioeconomic status. This raises the problem of disproportionate cultural levels and social inequity regarding access to healthcare services.

Almost all patients involved in this study immediately undergone radical hysterectomy in our hospital unit after being diagnosed with endometrial carcinoma on an endometrial biopsy. The minority of untreated patients as well as those without preoperative investigations in our clinic addressed other sanitary units. It is important to note that the lack of continuity in diagnostic investigations and treatment as well as the dispersion of severe cases between different sanitary units is detrimental not only to the patient but also to the healthcare system, implying high costs and the impossibility to centralize and follow up medical data.

In our study, almost one-quarter (24\%) of the women who have undergone radical hysterectomy due to endometrial carcinoma presented endometrial intraepithelial neoplasia (EIN) on a previous biopsy. As literature suggests, these cases are probably concomitant occurrences of endometrial carcinoma, because the cancer was most likely already present in the patient when EIN was diagnosed but it was missed due to sampling error [14].

Treatment for endometrial carcinoma varies with the risk for persistent or recurrent disease after surgical intervention. While in low-risk diseases no further treatment is generally recommended, women with high or highly-intermediate risk endometrial carcinoma must benefit from adjuvant therapy in order to lower the chances of recurrence. Adjuvant treatment includes chemotherapy and radiation therapy which can be delivered as vaginal brachytherapy or external beam radiation therapy.

In conclusion, the high amount of female patients presenting to the University Emergency Hospital in Bucharest for abnormal uterine bleeding facilitated the development of a vast experience involving endometrial pathology and its interdisciplinary correlations.

In our opinion and in accordance with the current healthcare tendency, transvaginal ultrasound remains the best cost-effective method for investigating postmenopausal abnormal uterine bleedings which must be subjected to endometrial biopsy, therefore tremendously reducing the amount of useless invasive maneuvers.

Our findings indicate that almost one-quarter of the patients diagnosed preoperatively with endometrial intraepithelial neoplasia may present endometrial carcinoma upon final examination of the hysterectomy specimen. Therefore, rigorous sampling is a major factor in effective detection of incipient invasive carcinoma or physically small and/or focal areas of endometrial intraepithelial neoplasia.

Precise evaluation of the tumor type in endometrial cancers is one of the most frequent issues encountered in endometrial biopsies. Accurate diagnosis and distinction between type I and type II endometrial carcinoma is of critical importance for proper surgical planning and and further clinical management.

We undersign, certificate that the procedures and the experiments we have done respect the ethical standards in the Helsinki Declaration of 1975, as revised in 2000 (5), as well as the national law.

\section{References}

1. SGO Clinical Practice Endometrial Cancer Working Group, Burke WM, Orr J, Leitao 
M, Salom E, et al. (2014) Endometrial cancer: a review and current management strategies: Part I. Gynecol Oncol 134: 385-392. [Crossref]

2. Colombo N, Preti E, Landoni F, Carinelli S, Colombo A, et al. (2011) Endometria cancer: ESMO Clinical Practice Guidelines for diagnosis, treatment and follow-up. Ann Oncol 22: vi35-vi39. [Crossref]

3. Bohîlțea RE, Ancăr V, Cirstoiu MM, Rădoi V, Bohîlțea LC, et al. (2015) Project for the National Program of Early Diagnosis of Endometrial Cancer Part I. J Med Life 8: 305-314. [Crossref]

4. Nucci MR, Esther O (2009) Gynecologic Pathology, Elsevier Churchill Livingstone, ISBN: 978-0-443-06920-8: 233-259.

5. Emons G, Beckmann MW, Schmidt D, Mallmann P; Uterus commission of the Gynecological Oncology Working Group (AGO) (2015) New WHO Classification of Endometrial Hyperplasias. Geburtshilfe Frauenheilkd 75: 135-136. [Crossref]

6. Cancer Genome Atlas Research Network, Kandoth C, Schultz N, Cherniack AD, Akbani R, et al. (2013) Integrated genomic characterization of endometrial carcinoma. Nature 497: 67-73. [Crossref]

7. Hendrickson M, Ross J, Eifel P, Martinez A, Kempson R (1982) Uterine papillary serous carcinoma: a highly malignant form of endometrial adenocarcinoma. Am J Surg Pathol 6: 93-108. [Crossref]
8. Bokhman JV (1983) Two pathogenetic types of endometrial carcinoma. Gynecol Oncol 15: 10-17. [Crossref]

9. Brinton LA, Hoover RN (1993) Estrogen replacement therapy and endometrial cancer risk: unresolved issues. The Endometrial Cancer Collaborative Group. Obstet Gynecol 81: 265-271. [Crossref]

10. Grady D, Gebretsadik T, Kerlikowske K, Ernster V, Petitti D (1995) Hormone replacement therapy and endometrial cancer risk: a meta-analysis. Obstet Gynecol 85: 304-313. [Crossref]

11. Weiderpass E, Adami HO, Baron JA, Magnusson C, Lindgren A, et al. (1999) Use of oral contraceptives and endometrial cancer risk (Sweden). Cancer Causes Control 10: 277-284. [Crossref]

12. Grimes DA, Economy KE (1995) Primary prevention of gynecologic cancers. Am J Obstet Gynecol 172: 227-235. [Crossref]

13. Cohen I (2004) Endometrial pathologies associated with postmenopausal tamoxifen treatment. Gynecol Oncol 94: 256-266. [Crossref]

14. Mutter GL, Prat J (2014) Pathology of the Female Reproductive Tract. (3rd Edn). Elsevier. Endometrial Adenocarcinoma: 370-401.

15. Kurman RJ, Carcangiu ML, Herrington CS, Young RH (2014) WHO Classification of Tumours of Female Reproductive Organs. (4th edn). International Agency for Research on Cancer, Lyon. p123, ISBN: 978-92-832-2435-8.

Copyright: (C) 2016 Georgescu TA. This is an open-access article distributed under the terms of the Creative Commons Attribution License, which permits unrestricted use, distribution, and reproduction in any medium, provided the original author and source are credited. 